\title{
UCRL-CONF-221183
}

LAW RENCE LIVERMORE N A T IO N A L LABORATORY

\section{High Magnetic field generation for laser-plasma experiments}

B. B. Pollock, D. H. Froula, P. F. Davis, J. S. Ross, S. Fulkerson, J. Bower, J. Satariano, D. Price, S. H. Glenzer

May 7, 2006

High Temperature Plasma Diagnostics Williamsburg, VA, United States May 7, 2006 through May 11, 2006 
This document was prepared as an account of work sponsored by an agency of the United States Government. Neither the United States Government nor the University of California nor any of their employees, makes any warranty, express or implied, or assumes any legal liability or responsibility for the accuracy, completeness, or usefulness of any information, apparatus, product, or process disclosed, or represents that its use would not infringe privately owned rights. Reference herein to any specific commercial product, process, or service by trade name, trademark, manufacturer, or otherwise, does not necessarily constitute or imply its endorsement, recommendation, or favoring by the United States Government or the University of California. The views and opinions of authors expressed herein do not necessarily state or reflect those of the United States Government or the University of California, and shall not be used for advertising or product endorsement purposes. 


\title{
High magnetic field generation for laser-plasma experiments
}

B. B. Pollock, ${ }^{*}$ D. H. Froula, P. F. Davis ${ }^{\dagger}$ J. S. Ross, S. Fulkerson, J. Bower, J. Satariano, D. Price, and S. H. Glenzer Lawrence Livermore National Laboratory, University of California, P.O. Box 808, Livermore, California 94551

(Date textdate; Received textdate; Revised textdate; Accepted textdate; Published textdate)

\begin{abstract}
An electromagnetic solenoid was developed to study the effect of magnetic fields on electron thermal transport in laser plasmas. The solenoid, which is driven by a pulsed power system suppling $30 \mathrm{~kJ}$, achieves magnetic fields of $13 \mathrm{~T}$. The field strength was measured on the solenoid axis with a magnetic probe and optical Zeeman splitting. The measurements agree well with analytical estimates. A method for optimizing the solenoid design to achieve magnetic fields exceeding $20 \mathrm{~T}$ is presented.
\end{abstract}

PACS numbers:

\section{INTRODUCTION}

Magnetic fields play a significant role in the physical behavior of plasmas relevant to Inertial Confinement Fusion (ICF), such as those produced at the National Ignition Facility (NIF)[1]. Specifically, magnetic fields effect electron heat transport within the plasma[2]. The properties of heat transport are of particular importance in understanding the spatial temperature dependence in ICF plasmas[3].

In 1933 Francis Bitter developed an electromagnet made from copper discs[4]. Each disc is a small (5 mm inner radius) circular ring with a 33 degree cutout. An insulating material was cut to the same dimension as the copper, and the solenoid was assembled in a stair-step fashion. Each disc was isolated from the next by an insulator, except in the region of the cutout. Electrical contact was made by tightly compressing the coil, allowing current to flow in the loops established by the overlapping regions and producing magnetic fields exceeding $20 \mathrm{~T}$.

In order to investigate the inhibition of electron heat transport in laser-produced plasmas, magnetic fields greater than $10 \mathrm{~T}$ are necessary $[5,6]$. While the conventional Bitter electromagnet is capable of producing such fields, the operating environment of laser experiments demands that numerous modifications be made. Two modified Bitter electromagnets were constructed in series, and access ports allow us to measure the local high magnetic fields, field diagnostics, propagate the laser beams, and insert the gas jet (Figure 1). The gas injected by the jet into the solenoid requires isolation of the high currents. We present an electromagnet design that has produced successful results in a laser-produced gas jet plasma at field strength of $13 \mathrm{~T}$.

The laser experiment performed at the Janus Laser Facility, Lawrence Livermore National Laboratory, em-

\footnotetext{
* School of Engineering and Computer Science, University of the Pacific, Stockton, CA 95211

${ }^{\dagger}$ Department of Physics, University of British Columbia, Vancou-
} ver, Canada, V2T 6W9

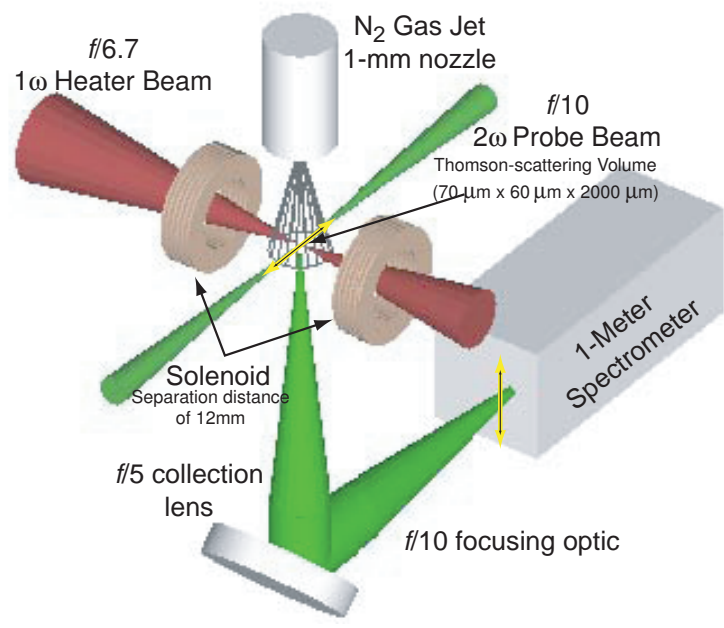

FIG. 1: The schematic for the laser setup is shown. A $12 \mathrm{~mm}$ gap between the coils allows access to the region with high magnetic fields. The system has been optimized to provide high fields directly between the coils where the laser beams cross.

ployed a gas jet to deliver $N_{2}$ gas that is heated by a high power (1 ns) laser beam with $100 \mathrm{~J}$ of $1 \omega(1053 \mathrm{~nm})$ light in the presence of an external magnetic field. Imaging Thomson Scattering[7] with $500 \mathrm{~mJ}$ of $2 \omega$ light is used to spatially resolve the electron temperature profile perpendicular to the laser beam $[8,9]$. A Rogowski $d I / d t$ probe measures the current through the solenoid, and is calibrated to determine the magnetic field strength. It was found that when fields above $10 \mathrm{~T}$ are applied along the laser beam axis the electron thermal transport and the peak electron temperature are strongly affected[9]. These results have demonstrated that the present solenoid design allows controlled laser-plasma experiments with a known magnetic field strength.

Section II of this paper discusses the magnet used in the laser experiment, including a description of the solenoid construction and the pulse power system used to drive the high magnetic fields. Section III presents a general prescription for designing and optimizing the solenoid. Section IV summarizes our results. 


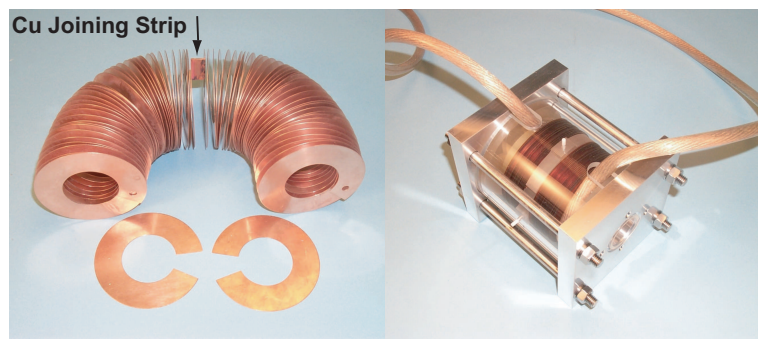

FIG. 2: (a) The coils are constructed by welding the ends of each disc. Two coils are separated by a $12 \mathrm{~mm}$ gap. (b) The coils are cast in high voltage epoxy for strength and to isolate all electrical surfaces from the experimental environment.

\section{MAGNETIC FIELD GENERATION}

\section{A. Solenoid Construction}

Figure 2 shows the coil parts and the assembled solenoid used in the laser experiment. The magnet consists of 2 coils, each having 41 discs $0.5 \mathrm{~mm}$ thick with $2.3 \mathrm{~cm}$ inner and $4.6 \mathrm{~cm}$ outer radii. A 33 degree wedge was cut in each disc, and neighboring discs were buttwelded together forming continuous coils (Figure 2). Between each disc a $127 \mu \mathrm{m}$ thick Kapton insulator was inserted. These were arranged with overlapping ends to prevent arcing between neighboring discs. The two identical 41 disc coils were joined by a strip of copper spanning a $12 \mathrm{~mm}$ gap between them, as illustrated in Figure 2a. Electric cabling is bolted to each end of the solenoid to supply current.

The experiment takes place in a vacuum of order $10^{-4}$ Torr. At shot time, the effective pressure inside the solenoid increases to about 1 Torr as the gas jet is fired $20 \mathrm{~ms}$ prior to the laser. Under these conditions, the electrical break-down potential is very low. Therefore, great care was taken to shield all conducting surfaces from the gas. The solenoid was placed inside a 4.5 inch outside diameter lucite shell, with a 1 inch inner diameter lucite cylinder inserted through the bore (the lucite wall thickness is 0.25 inches). Aluminum endcaps with 0.25 inch stainless steel rods hold the solenoid together, and Emerson and Cumming Stycast 1266 high voltage epoxy (500 kV/mm electrical standoff) is poured to fill the lucite shell as shown in Figure 2b. Optical ports are then machined in the central gap to allow diagnostic access.

\section{B. Pulse Power System}

\section{LRC Pulse Power Circuit}

Figure 3 shows the schematic for the pulse power system that delivers current to the magnet. Three capacitors $(\mathrm{C}=48 \mu \mathrm{F})$ are wired in parallel; two knife switches allow the selection of one, two, or all three capacitors. A resistor $(\mathrm{R}=1.5 \Omega)$ is placed in series with each capacitor

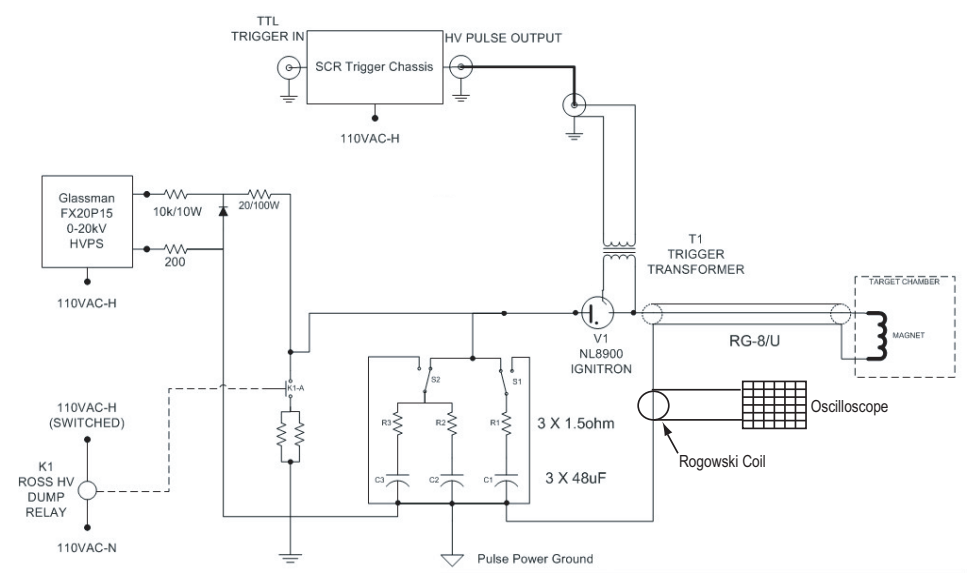

FIG. 3: The schematic for the pulse power system used to drive the solenoid. At maximum charge and with all three capacitors engaged $28.8 \mathrm{~kJ}$ are produced.

to damp the system. The current through the solenoid (inductor) is given by an LRC differential equation.

In our case $L>\frac{1}{4} C R^{2}$, so the system is weakly damped and may be described by

$$
I(t)=\frac{V_{0} e^{-t / \tau}}{\omega L} \sin (\omega t)
$$

where $\omega=\sqrt{\frac{1}{L C}-\left(\frac{R}{2 L}\right)^{2}}, \tau=\frac{2 L}{R}, V_{0}$ is the initial charge voltage, and $\mathrm{L}=140 \mu \mathrm{H}$ as measured with a $\mathrm{kHz}$ inductance meter.

The current (I) through the system is measured with a Rowgowski $d I / d t$ probe on the cable returning to ground in the pulse power capacitor bank. When all three capacitors are fully charged $\left(V_{0}=20 \mathrm{kV}\right)$, the system delivers a peak current of $\mathrm{I}=13 \mathrm{kA}$. The measured current agrees well with Equation 1 for $\mathrm{C}=144 \mu \mathrm{F}, \mathrm{L}=144 \mu \mathrm{H}$, and $\mathrm{R}$ $=662 \mathrm{~m} \Omega$, as shown in Figure 4. These are reasonable values given approximately $5 \mu \mathrm{H}$ of system inductance, $10 \mathrm{~m} \Omega$ of system resistance, $150 \mathrm{~m} \Omega$ of solenoid resistance, and negligible system capacitance.

\section{Capacitor Bank}

The desired charge voltage is set on a Glassman FX20P15 High Voltage Power System capable of supplying up to 20kV. A Ross High Voltage Dump Relay (k1) prevents the capacitors from charging or retaining charge when the dumps are engaged. The switching mechanism that allows current to flow between the bank and the solenoid is an NL8900 ignitron. An SCR Trigger Chassis takes in a TTL trigger and produces a high voltage pulse, which initiates the switching of the ignitron. The trigger transformer isolates the SCR from the pulse power system. 


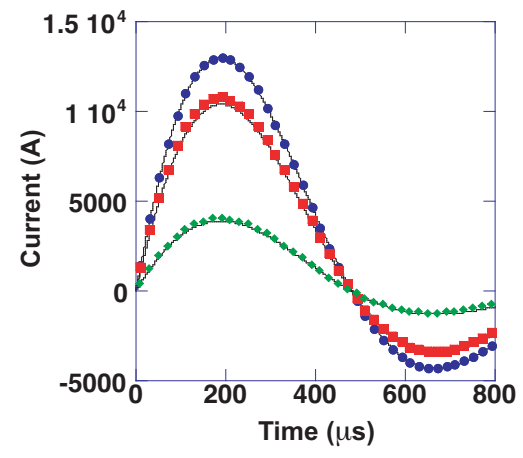

FIG. 4: Current traces from time-integrated Rogowski signals compared with calculations using Equation 1 for $V_{0}=20 \mathrm{kV}$, $16 \mathrm{kV}$, and $6 \mathrm{kV}$.

\section{Field Characterization}

\section{Pickup Probe}

To characterize the magnetic field along the axis of the solenoid a 10 turn, $1.5 \mathrm{~mm}$ diameter pickup probe was constructed and calibrated. Figure 5a shows the measured magnetic field strength between the coils $(\mathrm{z}=0)$ for a maximum capacitor charge of $V_{0}=20 \mathrm{kV}$ as compared with the results of Equation 2. Figure 5b shows the peak magnetic field recorded along the solenoid axis for a constant capacitor charge $\left(V_{0}=1 \mathrm{kV}\right)$.

The magnetic field produced along the axis of the solenoid $(\mathrm{z})$ is given by[10]

$$
\begin{gathered}
B(t, z)=I(t) G(z)=\frac{I(t) \mu_{0} N}{4 l}\left(\frac{z+l+\frac{d}{2}}{\sqrt{r^{2}+\left(z+l+\frac{d}{2}\right)^{2}}}-\right. \\
\left.\frac{z+\frac{d}{2}}{\sqrt{r^{2}+\left(z+\frac{d}{2}\right)^{2}}}+\frac{z-\frac{d}{2}}{\sqrt{r^{2}+\left(z-\frac{d}{2}\right)^{2}}}-\frac{z-l-\frac{d}{2}}{\sqrt{r^{2}+\left(z-l-\frac{d}{2}\right)^{2}}}\right)
\end{gathered}
$$

where $r=2.3 \mathrm{~cm}$ is the inner disc radius, $d=12 \mathrm{~mm}$ is the distance between the coils, $N=72$ is the total number of turns, and $l=2.26 \mathrm{~cm}$ is the length of each coil. Figure $5 \mathrm{~b}$ compares the calculated magnetic field profiles with the measurements.

\section{Zeeman Splitting}

The magnetic field produced between the coils is measured with Zeeman splitting through observing a split in atomic energy levels. Figure 6 shows the measured Zeeman spectrum for a $V_{0}=16 \mathrm{kV}$ charge. The field strength increases linearly with increasing current (Figure 7), and the results are consistent with Equation 2 when substituting for the peak current calculated using Equation 1.
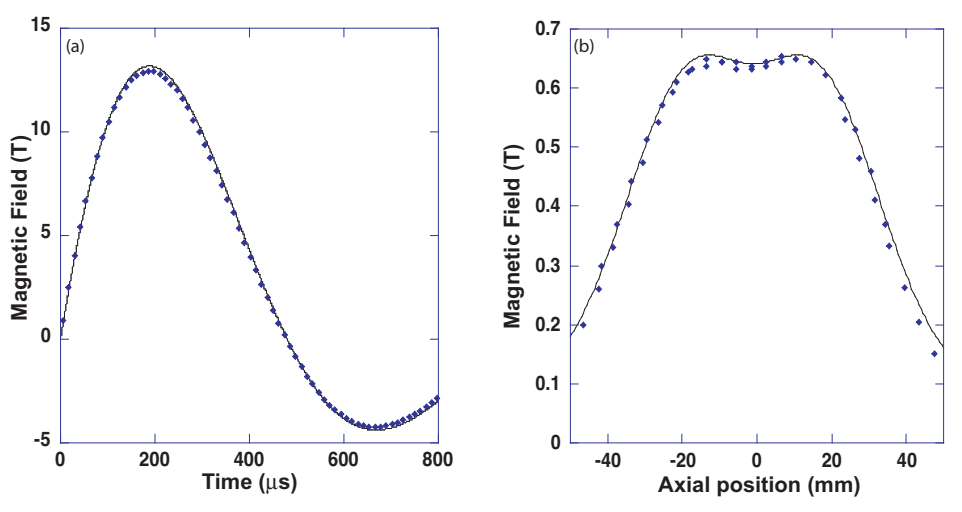

FIG. 5: (a) The magnetic field is measured at the center of the solenoid using a pick-up probe. (b) The magnetic field along the axis of the solenoid shows a maximum field inside the coils and a slight dip in the profile between the coils. Equation 2 is plotted on both plots using the parameters of our system.

The measurement is made using an Oriel Ne spectral lamp placed inside the solenoid. The emission is collected through the central gap with an $f / 5$ collection optic. The light is imaged through a $100 \mu \mathrm{m}$ slit into a 1-m spectrometer with an $f / 4$ lens. Using an 1800 lines/mm grating to measure a Neon line at $585.25 \mathrm{~nm}$ results in dispersion of $0.555 \mathrm{~nm} / \mathrm{mm}$. A $1 \mu \mathrm{s}$ gated CCD records the spectrum corresponding to the peak current through the solenoid.

An externally applied magnetic field will split degenerate atomic energy levels according to their angular momenta and the applied field strength. This corresponds to a shift in wavelength in the emission spectra given by

$$
\Delta \lambda=\frac{\lambda_{0}^{2} e B}{2 \pi c M}
$$

where $\lambda_{0}$ is the central wavelength, $\mathrm{e}$ is the charge of the electron, and $\mathrm{M}$ is the mass of the electron.

\section{MAGNETIC FIELD OPTIMIZATION}

The strength of the magnetic field produced by a solenoid is separable into the product of a time dependent current and a spatially dependent geometry factor which are coupled through the inductance. The inductance is determined by the geometry, which effects the current through a simple LRC system; therefore, the magnetic field between two coils can be optimized by varying the number of turns and the inner solenoid radius. The outer radius does not affect the inductance, and should be chosen with consideration to thermal mass and mechanical strength. We will show that for a given loop thickness and coil separation there are many combinations of the number of turns and the inner radius that give the same 


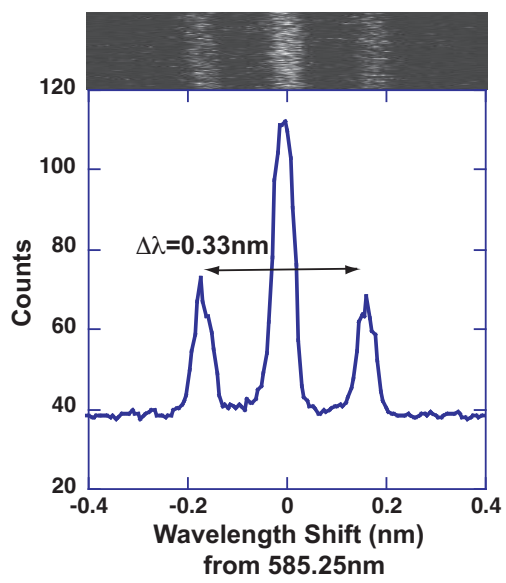

FIG. 6: Zeeman spectra for $V_{0}=16 \mathrm{kV}$. Shows a wavelength shift $(\Delta \lambda=0.33 \mathrm{~nm})$ corresponding to a $10 \mathrm{~T}$ magnetic field.

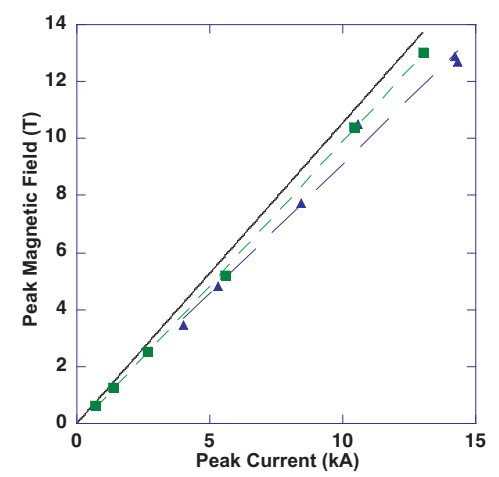

FIG. 7: The peak magnetic field is measured using a pick-up probe (squares), and by Zeeman splitting (triangles). Linear regressions of the data are included, and the data is compared to Equation 2 (solid line).

solenoid inductance, but there is one unique combination that maximizes the magnetic field. This section will determine the coil configuration which optimizes the field strength between the coils while keeping the field inside the solenoid below the yield point of the construction material.

\section{A. Solenoid Inductance}

Calculating the solenoid inductance as a function of the number of turns and the inner solenoid radius will allow us to optimize the current through the solenoid. The mutual inductance between any two current carrying loops of radius $r$ is given by[10]

$$
M_{A B}(k)=2 \mu_{0} k^{-1} r\left[\left(1-\frac{1}{2} k^{2}\right) K(k)-E(k)\right]
$$

where $k^{2}=4 r^{2}\left[4 r^{2}+c^{2}\right]^{-1}$, c is the distance between the centers of the loops, and $\mathrm{K}(\mathrm{k})$ and $\mathrm{E}(\mathrm{k})$ are the complete elliptic integrals of the first and second kind, respectively.

The inductance of a solenoid is the sum of all the mutual inductances between each pair of loops,

$$
L(N, r)=\sum_{A} \sum_{B} M_{A B}\left(k_{m}\right)=2 \sum_{m=1}^{N-1}\left((N-m) M\left(k_{m}\right)\right)
$$

where $k_{m}^{2}=4 r^{2}\left[4 r^{2}+(m c)^{2}\right]^{-1}, \mathrm{~N}$ is the total number of turns, $\mathrm{m}=\mathrm{B}-\mathrm{A}$, and $\mathrm{A}$ is less than $\mathrm{B}$. The magnet design requires a separation $(\mathrm{d})$ between the center two turns which modifies the sum in the following way

$$
\begin{aligned}
& L(N, r)=2 \sum_{m=1}^{\frac{N}{2}-1}\left((N-2 m) M\left(k_{m}\right)+\right. \\
& \left.m M\left(k_{m}^{*}\right)\right)+2 \sum_{m=\frac{N}{2}}^{N-1}(N-m) M\left(k_{m}^{*}\right)
\end{aligned}
$$

where $\left(k_{m}^{*}\right)^{2}=4 r^{2}\left[4 r^{2}+(c(m-1)+d)^{2}\right]^{-1}$. For the parameters in our experiment $(\mathrm{N}=72, \mathrm{r}=23 \mathrm{~mm}, \mathrm{~d}$ $=12 \mathrm{~mm}$, and $\mathrm{c}=627 \mu \mathrm{m}$ ) the inductance is calculated by Equation 6 to be $132 \mu \mathrm{H}$, which agrees well with the measured value of $140 \mu \mathrm{H}$.

\section{B. Magnetic Field Calculation}

The current through an LRC system is a function of the charge voltage, capacitance, resistance, and inductance. A critically damped LRC system produces a time dependent current,

$$
I(t)=\frac{V_{0}}{L(N, r)} t e^{-\frac{R t}{2 L(N, r)}}
$$

where the resistance is chosen such that $R^{2}=\frac{4 L}{C}$. Substituting the peak current generated by a critically damped LRC circuit normalized by the total stored en$\operatorname{ergy}\left(J_{\text {peak }}=\frac{I_{\text {peak }}}{\sqrt{E}}=\sqrt{\frac{2}{e^{2} L(N, r)}}\right)$ into Equation 2, the magnetic field between the coils $(\mathrm{z}=0)$ can be expressed,

$$
\begin{gathered}
H(N, r)=J_{\text {peak }} G(0)=\sqrt{\frac{2}{e^{2} L(N, r)}} \times \\
\frac{\mu_{0} N}{2 l}\left(\frac{l+\frac{d}{2}}{\sqrt{r^{2}+\left(l+\frac{d}{2}\right)^{2}}}-\frac{\frac{d}{2}}{\sqrt{r^{2}+\left(\frac{d}{2}\right)^{2}}}\right)
\end{gathered}
$$

where $H=\frac{B}{\sqrt{E}}$ is the normalized magnetic field strength and $E=\frac{1}{2} C V_{0}^{2}$ is the stored energy in the system. An optimal solenoid design is determined by maximizing Equation 8. 


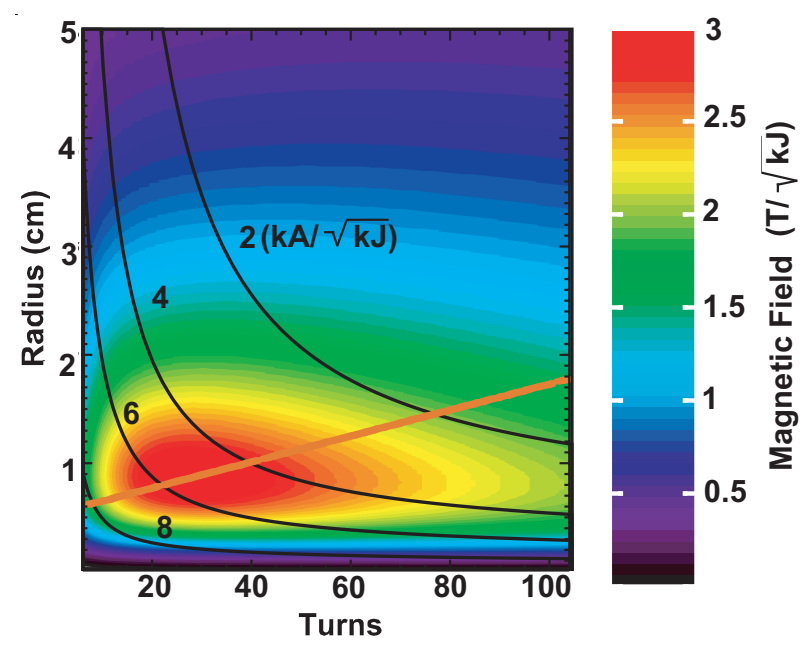

FIG. 8: A contour plot of Equation 8 showing the normalized magnetic field strength as a function of the number of turns and the radius of the solenoid. Also included are curves of constant current (black). The orange line passes through the maximum of Equation 8 and indicates the set of optimal designs when the material strength of the solenoid must be considered.

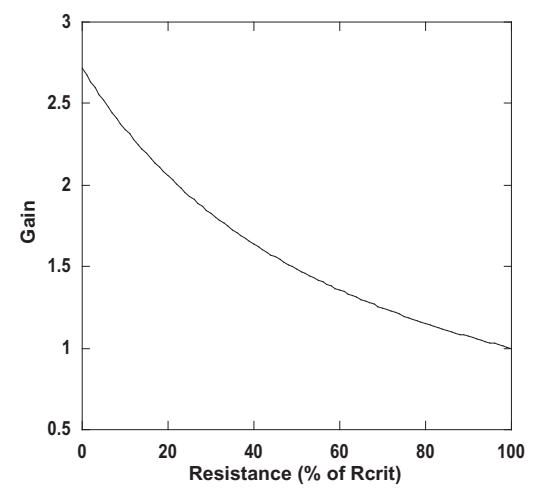

FIG. 9: Gain $\left(\frac{I(R)}{I\left(R_{\text {crititical }}\right)}\right)$ in the peak current is achieved by allowing the system resistance to be less than the critical damping value of $R=2 \sqrt{\frac{L}{C}}$. This relates directly to a gain in the magnetic field strength.

\section{Solenoid Optimization}

There are four geometrical parameters that determine the magnetic field strength produced by a solenoid: the coil separation, turn spacing, number of turns, and inner solenoid radius. This section will discuss optimizing the solenoid design in terms of these parameters, their influence on the inductance, and the yield point of the solenoid material (approximately $30 \mathrm{~T}$ for copper).

\section{Magnetic Field Strength}

Figure 8 is a contour plot of Equation 8 and gives the magnetic field as a function of radius and number of turns between the coils for a critically damped system with $\mathrm{c}=627 \mu \mathrm{m}, \mathrm{d}=12 \mathrm{~mm}$, and $4 \mu \mathrm{H}$ of system inductance. It shows the optimal solenoid design ( $\mathrm{N}=26$ turns, $\mathrm{r}=8.6 \mathrm{~mm}$ ), which requires a peak normalized current of $3.8 \mathrm{kA} / \sqrt{k J}$. On our system $(\mathrm{E}=28.8 \mathrm{~kJ})$ this corresponds to a maximum magnetic field of $B_{\text {peak }}(z=0)=16 \mathrm{~T}$ while producing a $\mathrm{B}=23 \mathrm{~T}$ field inside the solenoid. Increasing the current by either reducing the system resistance (allowing the system to be underdamped, resulting in a current gain given by Figure 9) or by increasing the stored energy will increase the optimal magnetic field strength. This optimization is valid until the magnetic pressure inside the solenoid exceeds the yield point of the construction material. For copper coils, this corresponds to a magnetic field of $30 \mathrm{~T}$.

The Lorentz force is given by $\mathbf{F}=\mathrm{q} \mathbf{v} \times \mathbf{B}$. The current in our application is azimuthal and the magnetic field is axial, so the force acts radially. In the case of an ideal solenoid this becomes $F=N 2 \pi r I B$; substituting $I=$ $\frac{B c}{\mu_{0}}$, where $\mathrm{c}$ is the turn thickness, and dividing by the inner surface area of the solenoid provides the magnetic pressure acting on the inner wall and can be calculated as $P=\frac{B^{2}}{\mu_{0}}$.

\section{Material Strength Considerations}

For a system that can produce fields large enough to exceed the yield point of the material used to construct the coils, the optimal magnetic field between the coils is not given by maximizing Equation 8. Figure 8 (black curves) shows that there are several combinations of the number of turns and radius that produce the same peak current; there is a single point on each curve that maximizes the geometry factor $(\mathrm{G}(\mathrm{z}))$ in Equation 8 and produces a maximum magnetic field (Fig. 8, Orange Line). This can be shown to follow the relation,

$$
r=0.11 N+5.81
$$

where $\mathrm{r}$ is in millimeters, and can be analytically verified in the limit of large numbers of turns. The optimal solenoid geometry will now correspond to a point on this line.

Figure 10 shows the strength of the magnetic field between the coils and the maximum field experienced inside the solenoid along the optimal line defined by Equation 9 . Scaling the stored energy so that the peak field inside the solenoid (dashed curve) is $30 \mathrm{~T}$ corresponds to $50 \mathrm{~kJ}$ of stored energy and a peak field between the coils (dotted curve) of $20 \mathrm{~T}$. This defines an optimum design for any critically damped system with a stored energy $\mathrm{E} \leq 50 \mathrm{~kJ}$. In order to access fields greater than $20 \mathrm{~T}$ between the 


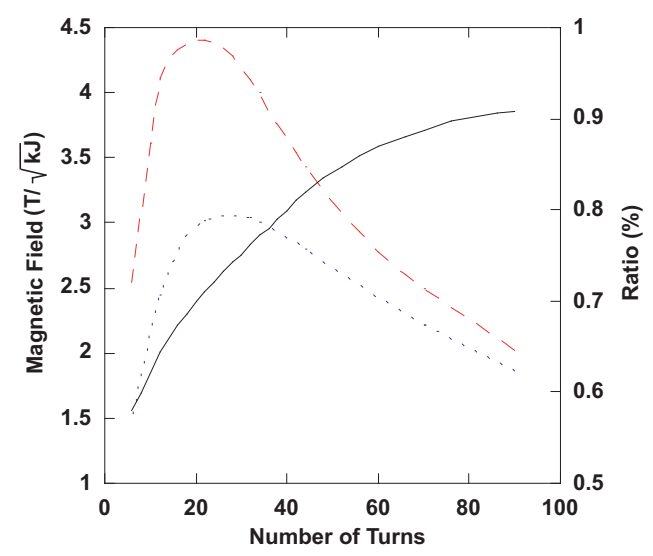

FIG. 10: Ratio (solid) of the magnetic field produced between the coils (dotted) to the maximum field inside the solenoid (dashed) along the optimal line defined by Equation 9. The selection of a ratio defines the system geometry and the maximum field possible between the coils.

coils the system must either be underdamped or capable of storing more than $50 \mathrm{~kJ}$. This forces the selection of a solenoid geometry providing a higher ratio of field strengths (Fig. 10 solid curve).

In this regime, our goal is to maximize the field strength between the coils without the field inside exceed- ing the yield point of the construction material. Selecting a ratio of field strengths defines the system geometry and the maximum magnetic field possible between the coils. The required stored energy can then be determined such that the field inside the solenoid reaches the yield point; the stored energy can be reduced by reducing the resistance of the LRC circuit, thereby increasing the the current.

\section{CONCLUSION}

A solenoid is constructed from two coils in series with a gap between them to allow access for a laser plasma interaction experiment. The field between the coils has been designed to be greater than $10 \mathrm{~T}$ using a stored energy of $30 \mathrm{~kJ}$. An analytical model has been presented that agrees well with the magnetic field measurements along the solenoid axis.

We have expanded this model to show that for systems with less than $50 \mathrm{~kJ}$ of stored energy there is an optimal solenoid design, limited by the material strength of copper, providing a $20 \mathrm{~T}$ field between the coils. To achieve fields greater than this threshold, the optimal design is chosen by the total stored energy.

We would like to thank the Janus Laser Crew for their outstanding support during our campaign. This work was performed under the auspices of the U.S. Department of Energy by the Lawrence Livermore National Laboratory under Contract No. W-7405-ENG-48.
[1] J. D. Lindl, P. A. Amendt, R. L. Berger, S. G. Glendinning, S. H. Glenzer, S. W. Haan, R. L. Kauffman, O. L. Landen, and L. J. Suter, Phys. Plasmas 11, 339 (2004).

[2] P. Nicolai, M. Vandenboomgaerde, B. Canaud, and F. Chaigneau, Phys. Plasmas 7, 4250 (2000).

[3] S. H. Glenzer, W. E. Alley, K. G. Estabrook, J. S. D. Groot, M. G. Haines, J. H. Hammer, J. P. Jadaud, B. J. MacGowan, J. D. Moody, W. Rozmus, et al., Phys. Plasmas 6 (1999).

[4] F. Bitter, Rev. Sci. Instrum. 10, 373 (1939).

[5] A. A. Offenberger and N. H. Burnett, Can. J. Phys. 53, 1360 (1975).

[6] N. H. Burnett and A. A. Offenberger, J. Appl. Phys. 45, 2155 (1974).
[7] J. S. Ross, D. H. Froula, A. Mackinnon, C. Sorce, S. H. Glenzer, W. Armstrong, R. Bahr, R. Huff, and K. Thorp, Submitted to RSI (2006).

[8] G. Gregori, S. H. Glenzer, J. Knight, C. Niemann, D. Price, D. H. Froula, M. J. Edwards, R. P. J. Town, W. Rozmus, A. Brantov, et al., Phys. Rev. Lett. 92 (2004).

[9] D. H. Froula, P. F. Davis, B. B. Pollock, L. Divol, J. S. Ross, J. Edwards, R. P. J. Town, D. Price, S. H. Glenzer, A. A. Offenberger, et al., Submitted to PRL (2006).

[10] W. R. Smythe, Static and Dynamic Electricity (Hemisphere Publishing Corporation, New York, 1989), 3rd ed. 